\title{
Photodynamic inheritance from methylene blue to carbon dots against reduction, aggregation, and DNA interference
}

\author{
Ning $\mathrm{Xu}^{1}$, Quanyong $\mathrm{Gu}^{1}$, Jianjun $\mathrm{Du}^{1,2^{*}}$, Haoying $\mathrm{Ge}^{1}$, Saran Long ${ }^{1,2}$, Wen Sun ${ }^{1,2}$, Jiangli Fan ${ }^{1,2}$ and \\ Xiaojun Peng
}

\begin{abstract}
Photodynamic therapy (PDT) is a treatment that uses light-sensitive drugs and a light source to destroy cancer cells. Methylene blue (MB) is an efficient photosensitizing agent that has been widely used in PDT. However, MB suffers from the hypochromic effect that is caused by self-aggregation and DNA binding in vivo. It is also easily reduced to ineffective leucomethylene blue in the hypoxic environments surrounding solid tumors. In this work, we prepared $\mathrm{MB}$ carbon dots (MB-CDs) using $\mathrm{MB}$ as the carbon source. The MB-CDs not only inherit the PDT capabilities of MB, but also demonstrate good biocompatibility and low toxicity. Importantly, MB-CDs demonstrate excellent resistance to interference from reduction, aggregation, and DNA interactions. The MB-CDs exhibited satisfactory PDT activities both in vitro and in vivo. The tumor sizes were reduced to below $20 \%$ of their original volumes when irradiated by a $590-\mathrm{nm}$ lightemitting diode source.
\end{abstract}

Keywords: carbon dots, photodynamic therapy, methylene blue, low toxicity, resistance to interference.

\section{INTRODUCTION}

Photodynamic therapy (PDT) is a novel treatment strategy that enables good control over spatial and temporal resolution [1-6]. Compared with conventional tumor treatments, PDT avoids the possible risks of organ/neurological damage during surgery, drug resistance during chemotherapy, and the side effects of exposure to radiotherapy. Therefore, PDT has been successfully applied to tumors, such as colonic tumors, basal cell carcinomas, Kaposi's sarcomas, and melanomas. Nevertheless, highperformance photosensitizers are highly needed to improve PDT [7-15].
Methylene blue (MB), an efficient broad-spectrum photosensitizer, strongly absorbs light at $660 \mathrm{~nm}$ in homogeneous media [16,17]. Although $\mathrm{MB}$ is weakly fluorescent (with a quantum yield of about 0.04), it has the singlet oxygen quantum yield $(\sim 0.57)$ in solution, and has been successfully used to treat cutaneous cancers, melanomas, and tumors in subcutaneous locations $[18,19]$. MB is easily reduced to leucomethylene blue (leucoMB, an uncharged colorless compound) in vivo by reductant (nicotinamide adenine dinucleotide (NADH), glutathione, etc.) or reducing enzymes (e.g., diaphorase) in the hypoxic environments surrounding solid tumors [20]. Colorless leucoMB fails in PDT and is rapidly excreted. Despite the spontaneous re-oxidation of some residual leucoMB to $\mathrm{MB}$, leucoMB consumes more $\mathrm{O}_{2}$, which exacerbates the hypoxic condition [21]. In addition, $\mathrm{MB}$ tends to form dimers due to $\pi-\pi$ stacking, resulting in blue-shifted absorption [22]. Moreover, it is important to note that $\mathrm{MB}$ has been reported to interact with DNA, leading to a hypochromic effect that limits PDT efficiency $[23,24]$. Although MB is efficient in vitro, the above-stated disadvantages impede its efficient therapy of solid tumors.

Lately, carbon dots (CDs) have attracted a great deal of attention as promising photosensitizers, owing to their remarkable properties, including good biocompatibility, low toxicity, and light-selective activation [25-32]. CDs are prepared from a "bottom-up" approach, namely from small (bio)molecules via hydrothermal synthesis [33]. Fortunately, similarly to genetic processes, certain characteristics of the original carbon source are transferred to the down-stream CDs. For instance, Ge et al. [34] successfully prepared photosensitized graphene quantum

\footnotetext{
${ }^{1}$ State Key Laboratory of Fine Chemicals, Dalian University of Technology, Dalian 116024, China

${ }^{2}$ Ningbo Institute of Dalian University of Technology, Ningbo 315016, China

*Corresponding author (email: dujj@dlut.edu.cn)
} 
dots based on polythiophene derivatives; they exhibited a high ${ }^{1} \mathrm{O}_{2}$ quantum yield because both the $\Delta \mathrm{EST}(\mathrm{S} 1 \rightarrow \mathrm{T} 1)$ and $\triangle \mathrm{ETG}(\mathrm{T} 1 \rightarrow \mathrm{G})$ energy gaps were sufficient to generate ${ }^{1} \mathrm{O}_{2}$. Subsequently, He et al. [35] fabricated diketopyrrolopyrrole-based CDs that effectively inhibited tumor growth under laser irradiation $(540 \mathrm{~nm})$ both in vitro and in vivo. Recently, Jia et al. [36] further developed special $\mathrm{Mn}$-doped CDs from manganese(II) phthalocyanine, in which oxygen was generated by $\mathrm{H}_{2} \mathrm{O}_{2}$ via a Mn catalyst to promote photodynamic effects in hypoxic microenvironments.

Against this backdrop, we assumed that photosensitizer-derived CDs inherit the excellent PDT capabilities of the carbon source and simultaneously maintain the intrinsic stability of the CDs against the redox microenvironment in solid tumors. Furthermore, multi-charged functional groups (e.g., $-\mathrm{COO}^{-}$and $-\mathrm{NH}^{3+}$ ) on the CDs provide effective steric hindrance and electrostatic interactions that hinder self-aggregation and binding to DNA, respectively. As a proof of concept, we synthesized optimal MB-CDs using a one-step hydrothermal method (at a temperature of $180^{\circ} \mathrm{C}$ for $8 \mathrm{~h}$ ) with
$\mathrm{MB}$ as the carbon source, as shown in Scheme 1. As expected, the MB-CDs not only inherited the photodynamic capabilities of $\mathrm{MB}$, but also retained the excellent water solubility, photostability, and low toxicity inherent to CDs, and effectively killed cancer cells in vitro and in vivo. More importantly, the MB-CDs displayed a surprising resistance to reduction, aggregation, and interaction with DNA, which perfectly address key issues associated with $\mathrm{MB}$ in PDT.

\section{EXPERIMENTAL SECTION}

\section{Materials and instruments}

(3-(4,5)-Dimethylthiazol-2-yl)-3,5-diphenyltetrazolium bromide (MTT), 1,3-diphenylisobenzofuran (DPBF), and PEG-800 were obtained from Aladdin Co., Ltd. MB was obtained from Tianjin Bodi Chemical Co., Ltd. The phosphate buffer saline (PBS) and Dulbecco's modified Eagle medium (DMEM) were obtained from Gibco. The reactive oxygen species (ROS) detection kit DCFH-DA (2,7-dichlorodihydrofluorescein diacetate) and the Annexin V-fluorescein isothiocyanate (FITC) and propi-

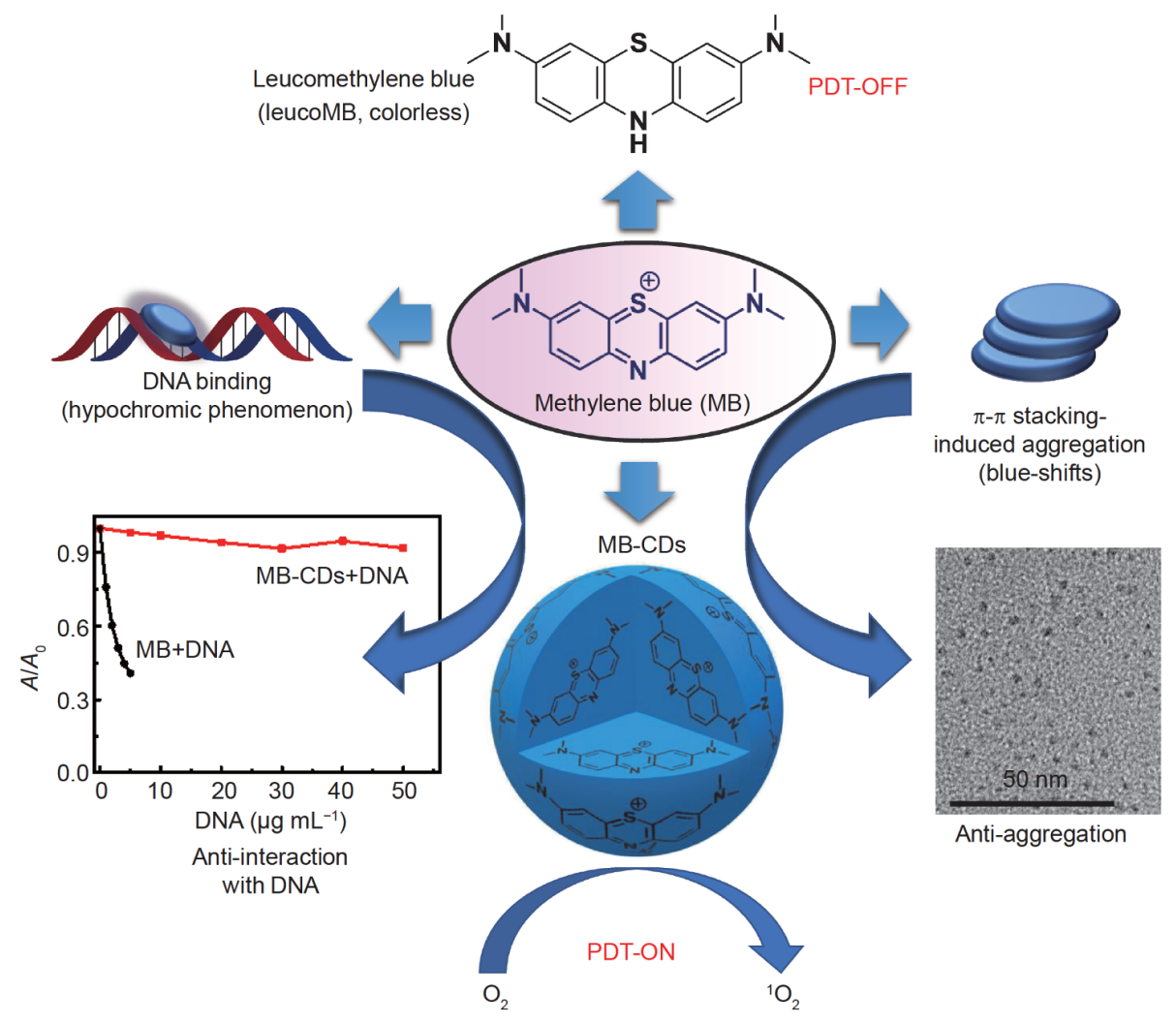

Scheme 1 Schematic showing the advantages of MB-CDs compared with MB. 
dium iodide (PI) apoptosis kits were purchased from Nanjing KeyGen Biotech. Co. Ltd. $\beta$-Nicotinamide adenine dinucleotide, reduced dipotassium salt (NADH), and diaphorase were purchased from Shanghai Yuanye BioTechnology Co. Ltd. All chemicals were used directly without further purification.

The ultraviolet-visible (UV-vis) spectra were recorded on an Agilent Technologies CARY 60 UV-vis spectrophotometer (serial no. MY1523004), whereas the fluorescent spectra were recorded on a Varian CARY Eclipse fluorescence spectrophotometer (serial no. MY15210003). The ultrapure water was provided by a Milli-Q system in all experiments. We used the Tecnai G2 F30 S-Twin microscope for transmission electron microscopy (TEM) analysis with an accelerating voltage of $300 \mathrm{kV}$. Furthermore, we used the SCALAB250Xi spectrometer for recording the X-ray photoelectron spectra. The confocal fluorescent images were collected on an Olympus FV3000 confocal microscope (Olympus, Japan), and the MTT experiment was performed on a Thermo Fisher Spectrophotometer 3020 .

\section{Preparation of MB-CDs}

We placed $15 \mathrm{mg} \mathrm{MB}$ and $15 \mathrm{mg}$ PEG-800 in a $25-\mathrm{mL}$ beaker, added $15 \mathrm{~mL}$ ultrapure water, and stirred well. We then transferred the solution into a $50-\mathrm{mL}$ Teflon autoclave and set it at $180^{\circ} \mathrm{C}$ for $8 \mathrm{~h}$. After the reaction bundle was formed, the reaction solution was cooled to room temperature. Then, the reaction solution was centrifuged in a centrifuge at $8000 \mathrm{rmin}^{-1}$ for $20 \mathrm{~min}$ to remove the larger particles. Then the upper liquid was passed through a $0.22-\mu \mathrm{m}$ filter to obtain the purple liquid. The filtrate was dialyzed in a 500-1000 molecular weight dialysis bag for $12 \mathrm{~h}$ to obtain the MB-CD solution. The dialyzed liquid was freeze-dried to obtain MBCD solid.

\section{Determination of singlet oxygen quantum yield}

We used MB as a reference and DPBF as a singlet oxygen trap to detect the singlet oxygen quantum yield $\left(\Phi_{\triangle}\right)$. The DPBF could be oxidized by ${ }^{1} \mathrm{O}_{2}$ to $o$-dibenzoylbenzene, leading to the absorbance decreases at $415 \mathrm{~nm}$. First, the DPBF concentration was adjusted in $3 \mathrm{~mL}$ dichloromethane (DCM) to ensure proper absorbance at $415 \mathrm{~nm}$. We then added the appropriate concentrations of $\mathrm{MB}$ or $\mathrm{MB}-\mathrm{CD}$ to ensure proper absorbance of the mixture at $590 \mathrm{~nm}$. The mixed solution was then irradiated by a 590 -nm light-emitting diode (LED) source up to $6 \mathrm{~min}$. The absorption of DPBF was tested at $415 \mathrm{~nm}$ in the presence of MB or MB-CDs. The $\Phi_{\triangle}$ was calcu- lated by the following equation with $\mathrm{MB}$ as a reference: $\Phi \wedge=\Phi_{\mathrm{MB}}\left(k_{\mathrm{PS}} \times F_{\mathrm{MB}}\right) /\left(k_{\mathrm{MB}} \times F_{\mathrm{PS}}\right) \times 100 \%$,

where $\Phi \wedge$ is the singlet oxygen quantum yield of MBCDs, $\Phi_{\mathrm{MB}}$ is 0.57 in DCM, representing singlet oxygen quantum yield of MB, $k$ is the slope at which the DPBF absorbance at $415 \mathrm{~nm}$ decreases with the increase of irradiation time, and $F$ is the correction factor $(F=1-$ $10^{-\mathrm{OD}}$ ), where $\mathrm{OD}$ is $\mathrm{UV}$ absorption value of compounds at $590 \mathrm{~nm}$.

\section{Detection of ROS generation in cell}

Human breast cancer cells (MCF-7) were seeded in a Petri dish containing DMEM with $10 \%$ fetal bovine serum (FBS) and incubated for $24 \mathrm{~h}$ in an incubator at $37^{\circ} \mathrm{C}$ with $5 \% \mathrm{CO}_{2}$ and $95 \%$ air. Then, $50 \mu \mathrm{g} \mathrm{mL}^{-1} \mathrm{MB}-\mathrm{CDs}$ and $5 \mu \mathrm{mol} \mathrm{L}{ }^{-1}$ DCFH-DA were added in the dish and incubated for $1 \mathrm{~h}$, and the cell dishes were washed twice with PBS and irradiated by a $561-\mathrm{nm}$ laser for $1 \mathrm{~min}$. The fluorescence signal of DCF was collected at $500-550 \mathrm{~nm}$ by Olympus FV3000 confocal microscope with a $488-\mathrm{nm}$ laser.

\section{Live/dead cells co-staining assay}

MCF-7 cells were seeded in a Petri dish and incubated for $24 \mathrm{~h}$ in a cell incubator. Then, $50 \mu \mathrm{g} \mathrm{mL}^{-1} \mathrm{MB}-\mathrm{CDs}$ was added to the dish and incubated for $1 \mathrm{~h}$. The experimental group dishes were exposed to a 590-nm LED source at $25 \mathrm{~mW} \mathrm{~cm}^{-2}$ for $15 \mathrm{~min}$; a group with MB-CDs but without irradiation was tested for comparison, and a group without any treatment served as the control. After 12-h incubation, the cells were further stained with the Calcein-AM/PI Double Stain Kit according to the instruction manual. Finally, the fluorescence images were captured by an Olympus FV3000 confocal microscope. The green channel (AM) was excited at $488 \mathrm{~nm}$, and the fluorescence signal at $500-550 \mathrm{~nm}$ was collected by $4 \times$ objective lens. The red channel (PI) was excited at $561 \mathrm{~nm}$, and the fluorescence signal at $590-650 \mathrm{~nm}$ was collected.

\section{Cytotoxicity assay}

The MTT was reduced to formazan crystal by mitochondrial dehydrogenase to evaluate the cytotoxicities of MB and MB-CDs. Firstly, the MCF-7 cells (4T1 cells) were equally seeded in a 96-well microplate with $1 \times$ $10^{5} \mathrm{~mL}^{-1}$ cells for each well. The 96-well microplates were incubated with $100 \mu \mathrm{L}$ DMEM (10\% FBS) for $24 \mathrm{~h}$ in an incubator. Secondly, the microplates were washed twice with PBS, and incubated for $1 \mathrm{~h}$ after treatment with 
different concentrations of $\mathrm{MB}(0,0.1,0.8,1.5$, $\left.2.0 \mu \mathrm{mol} \mathrm{L}^{-1}\right)$ and MB-CDs $\left(0,1,5,10,20,50 \mu \mathrm{g} \mathrm{mL}^{-1}\right)$, respectively. The microplates were exposed to $590-\mathrm{nm}$ or $660-\mathrm{nm}$ light with an average optical density of $25 \mathrm{~mW} \mathrm{~cm}^{-2}$ for $15 \mathrm{~min}$, respectively, and then incubated in a cell culture incubator for $12 \mathrm{~h}$. The microplates in the control group were placed in a dark environment. Thirdly, the $30 \mu \mathrm{mol} \mathrm{L}{ }^{-1} \mathrm{NADH}$ and $0.05 \mathrm{mg} \mathrm{mL}^{-1}$ diaphorase were added to microplates after being incubated with $\mathrm{MB}$ or MB-CDs for $1 \mathrm{~h}$, then incubated for $30 \mathrm{~min}$ before the above light and dark treatment. Cells cultured in medium without photosensitizer (PS) were used as the control, and six replicates were set for each group to eliminate contingency. After being incubated for $12 \mathrm{~h}$, the $100 \mu \mathrm{L}$ diluted MTT $\left(0.5 \mathrm{mg} \mathrm{mL}^{-1}\right.$ in DMEM) solution was added into each well and cultured for $4 \mathrm{~h}$ more at $37^{\circ} \mathrm{C}$ in the cell incubator. Finally, the medium was carefully washed to leave the formed crystals which were then dissolved in $100 \mu \mathrm{L}$ dimethyl sulfoxide (DMSO). The optical density was determined on a microplate reader (Thermo Fisher Scientific) by subtracting the absorbance of the free-blank volume at $490 \mathrm{~nm}$. The cell viability was calculated according to the following equation:

$$
\begin{aligned}
\text { Cell viability }= & \left(\mathrm{OD}_{\mathrm{CD}}-\mathrm{ODK}_{\mathrm{CD}}\right) / \\
& \left(\mathrm{OD}_{\text {control }}-\mathrm{ODK}_{\text {control }}\right),
\end{aligned}
$$

where $\mathrm{OD}_{\mathrm{CD}}$ is the absorbance at different MB-CD concentrations, $\mathrm{ODK}_{\mathrm{CD}}$ is the blank well absorbance, $\mathrm{OD}_{\text {control }}$ is the control well absorbance without treatment, and $\mathrm{ODK}_{\text {control }}$ is the DMSO absorbance.

\section{Application of PDT in 4T1 tumor-bearing mouse model}

All animals were tested in accordance with the Guide for the Care and Use of Laboratory Animal Resources and the National Research Council. Tests were approved by the Biology and Medical Ethics Committee of the Dalian University of Technology. The tumor model mice were established by injecting 4T1 cells into the right hind leg of $\mathrm{Balb} / \mathrm{c}$ female mice ( 6 weeks old). When the tumor size reached about $100 \mathrm{~mm}^{3}$, all tumor-bearing mice were divided into four groups $(N=3)$ : (I) injection of PBS; (II) injection of PBS with LED source irradiation $\left(590 \mathrm{~nm}, 50 \mathrm{~mW} \mathrm{~cm}^{-2}, 20 \mathrm{~min}\right)$; (III) injection of $\mathrm{MB}$ CDs $\left(50 \mu \mathrm{g} \mathrm{mL}^{-1}\right)$; (IV) injection of MB-CDs $\left(50 \mu \mathrm{g} \mathrm{mL}^{-1}\right)$ with LED source irradiation $(590 \mathrm{~nm}$, $\left.50 \mathrm{~mW} \mathrm{~cm}^{-2}, 20 \mathrm{~min}\right)$. The PDT effects were investigated by recording the tumor volumes and body weights of each group every $2 \mathrm{~d}$ for a total of $20 \mathrm{~d}$. The tumor volumes were tested and estimated by using the equation of $V=a \times b^{2} / 2$, where $V$ represents the tumor volume of mouse, and $a$ and $b$ represent the tumor length and width, respectively. The tumor-bearing mice were euthanized after the 20-d treatment, whereas the tumor and main organs (heart, liver, spleen, lung, and kidney) were collected and then fixed to buffered formalin. The hematoxylin and eosin (H\&E) staining was used for pathological analysis after paraffin sectioning.

\section{RESULTS AND DISCUSSION}

\section{Structural characterization of MB-CDs}

As shown in Fig. 1a, b, uniformly dispersed MB-CDs with an average diameter of $4.1 \mathrm{~nm}$ from 3.1 to $7.5 \mathrm{~nm}$ were observed by TEM and dynamic light scattering in water (Fig. S1). And high-resolution TEM (HR-TEM) observation shows the relevant lattice spacing of $0.21 \mathrm{~nm}$, which is consistent with the (100) facet of graphite. The zeta potential of MB-CDs was around $-3.63 \mathrm{mV}$ (Fig. S2). The elemental compositions and surface states of the MBCDs were examined by X-ray photoelectron spectroscopy (XPS). As shown in Fig. 1c, the typical C 1s, N 1s, O 1s, and $S 2$ p peaks were observed at 284.6, 399.4, 531.4, and $163.8 \mathrm{eV}$ with relative atomic percentages of $60.53 \%$, $13.11 \%, 22.36 \%$, and $4.00 \%$, respectively. The high-resolution $\mathrm{C} 1 \mathrm{~s}$ spectrum was divided into three typical peaks at 284.6, 285.9, and $288.0 \mathrm{eV}$ that correspond to $\mathrm{C}-\mathrm{C} / \mathrm{C}=\mathrm{C}, \mathrm{C}-\mathrm{N} / \mathrm{C}-\mathrm{O} / \mathrm{C}-\mathrm{S}$, and $\mathrm{C}=\mathrm{O}$ units, respectively (Fig. 1d) [37]. The $\mathrm{N}$ 1s spectrum (Fig. 1e) shows two peaks at 399.6 and $401.5 \mathrm{eV}$ that correspond to pyridinic and graphitic $\mathrm{N}$, respectively [38]. The high-resolution S 2 p spectrum revealed three peaks $(164.0,165.2$, and $168.4 \mathrm{eV}$ ) that correspond to $\mathrm{S} 2 \mathrm{p}_{3 / 2}$ and $\mathrm{S} 2 \mathrm{p}_{1 / 2}$ of the thiophene $S$ and oxidized S, respectively (Fig. 1f) [37,39]. The high-resolution $\mathrm{O}$ 1s spectrum showed the presence of $\mathrm{C}-\mathrm{OH}(531.3 \mathrm{eV})$ and $\mathrm{C}=\mathrm{O}(532.8 \mathrm{eV})$ units (Fig. S3) $[40,41]$. As expected, the core $\mathrm{MB}$ structure was retained during the synthesis of the MB-CDs, including the sulfur and nitrogen heteroatoms, which is critical for PDT performance.

\section{Spectral properties of MB-CDs}

The absorption spectrum of the MB-CDs shows broad absorption peaks in the $500-650 \mathrm{~nm}$ range that are mainly attributed to $n-\pi *(\mathrm{C}-\mathrm{N} / \mathrm{C}-\mathrm{S})$ transitions. The MB-CDs displayed maximum fluorescence absorption at around $590 \mathrm{~nm}$ in water (Fig. 2a). Interestingly, only the intensity of fluorescence emission at $620 \mathrm{~nm}$ changed significantly as the excitation wavelength was increased from 530 to $620 \mathrm{~nm}$ while keeping the emission wavelength the same (Fig. 2b). This excitation-independent 

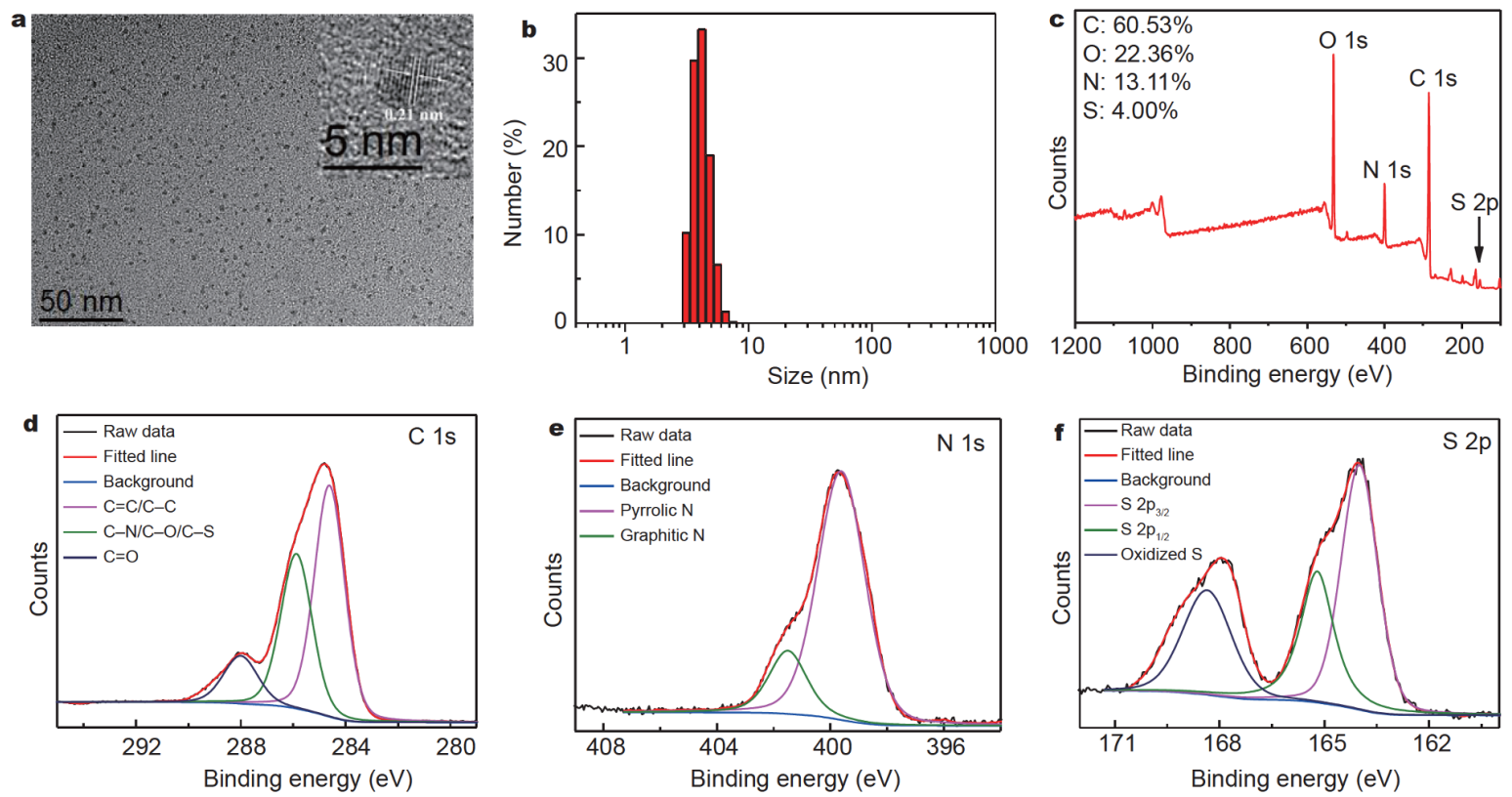

Figure 1 (a) TEM of MB-CDs (inset: HR-TEM image). (b) Dynamic light scattering of MB-CDs. (c) XPS spectrum of MB-CDs. (d) High-resolution XPS spectra of (d) C 1s, (e) N 1s, and (f) S 2p.

fluorescence behavior is related to various surface sites (surface state) and carbon core [33,42]. In addition, the MB-CDs were favorably dispersed and remained stable in water, PBS, and DMEM media (Figs S4 and S5). Compared with excitation wavelengths of $300-550 \mathrm{~nm}$ that were mostly used for CDs reported in the literature, we used a 590-nm LED light source that avoids background absorption (e.g., by hemoglobin) and penetrates deeper [43-46]. An excellent-performing photosensitizer should strongly absorb light, efficiently undergo intersystem crossing to generate the triplet state, and exhibit a long triplet lifetime, for facilitating interactions with other reactant molecules [47]. The nanosecond time-resolved transient absorption spectra (Fig 2c, d, and Fig. S6) indicate that the MB-CDs have inherited the photosensitization advantages of $\mathrm{MB}$ with a long triplet lifetime $(\tau=11.43 \mu \mathrm{s})$, which promotes effective photosensitization. ${ }^{1} \mathrm{O}_{2}$ efficiency was examined using DPBF as a ${ }^{1} \mathrm{O}_{2}$ trap. As shown in Fig. 2e, f, the absorbance of DPBF at $415 \mathrm{~nm}$ decreased sharply in the presence of the MB-CDs when irradiated by the 590-nm LED source for a prolonged time (Fig. S7). The singlet oxygen quantum yield of MB-CDs was calculated to be 0.24 using $\mathrm{MB}$ as a reference (Fig. S8), highlighting their strong potential for PDT.

Cell imaging and cytotoxicity experiments of MB-CDs MCF-7 cells were used to study the biocompatibility and cell penetrability of the MB-CDs in vitro experiments. MB-CDs quickly penetrated MCF-7 cells. The cells emitted a strong red fluorescence signal after incubation with the MB-CDs for $1 \mathrm{~h}$ (Fig. 3a). In addition, we also used $4 \mathrm{~T} 1$ cells for fluorescence imaging. They showed a red fluorescence signal after being incubated with the MB-CDs for $1 \mathrm{~h}$ (Fig. S9). Relative cell viabilities, which indicate the PDT effect, were determined using the standard MTT assay. As shown in Fig. 3b, MCF-7 cells were first incubated with $\mathrm{MB}-\mathrm{CD}$ of different concentrations $\left(0,1,5,10,20\right.$, and $\left.50 \mu \mathrm{g} \mathrm{mL}^{-1}\right)$ without being irradiated. We obtained almost $100 \%$ cell viability (black bars) without any obvious morphological changes after $12 \mathrm{~h}$ of incubation. This result indicates negligible cytotoxicity in the absence of light. The cell viability clearly decreased when MB-CD concentration increased, and cells were exposed to a 590-nm LED source (25 $\left.\mathrm{mW} \mathrm{cm}^{-2}, 15 \mathrm{~min}\right)$ (blank bars). This demonstrates the great ability of MB-CDs to kill cancer cells. The high photodynamic impact of MB-CDs is also demonstrated by MTT data from the 4 T1 cells (Fig. S10).

We used DCFH-DA, a commercial fluorescent ${ }^{1} \mathrm{O}_{2}$ indicator, to show $\mathrm{O}_{2}$ production by the MB-CDs in the cells [48]. Fig. 3c reveals that MCF-7 cells incubated with MB-CDs and DCFH-DA exhibit strong green fluorescence when exposed to a 561-nm laser. This is mainly due to the oxidation of dichlorolfuorescein. A weak fluorescence signal was observed in the absence of MB-CDs 

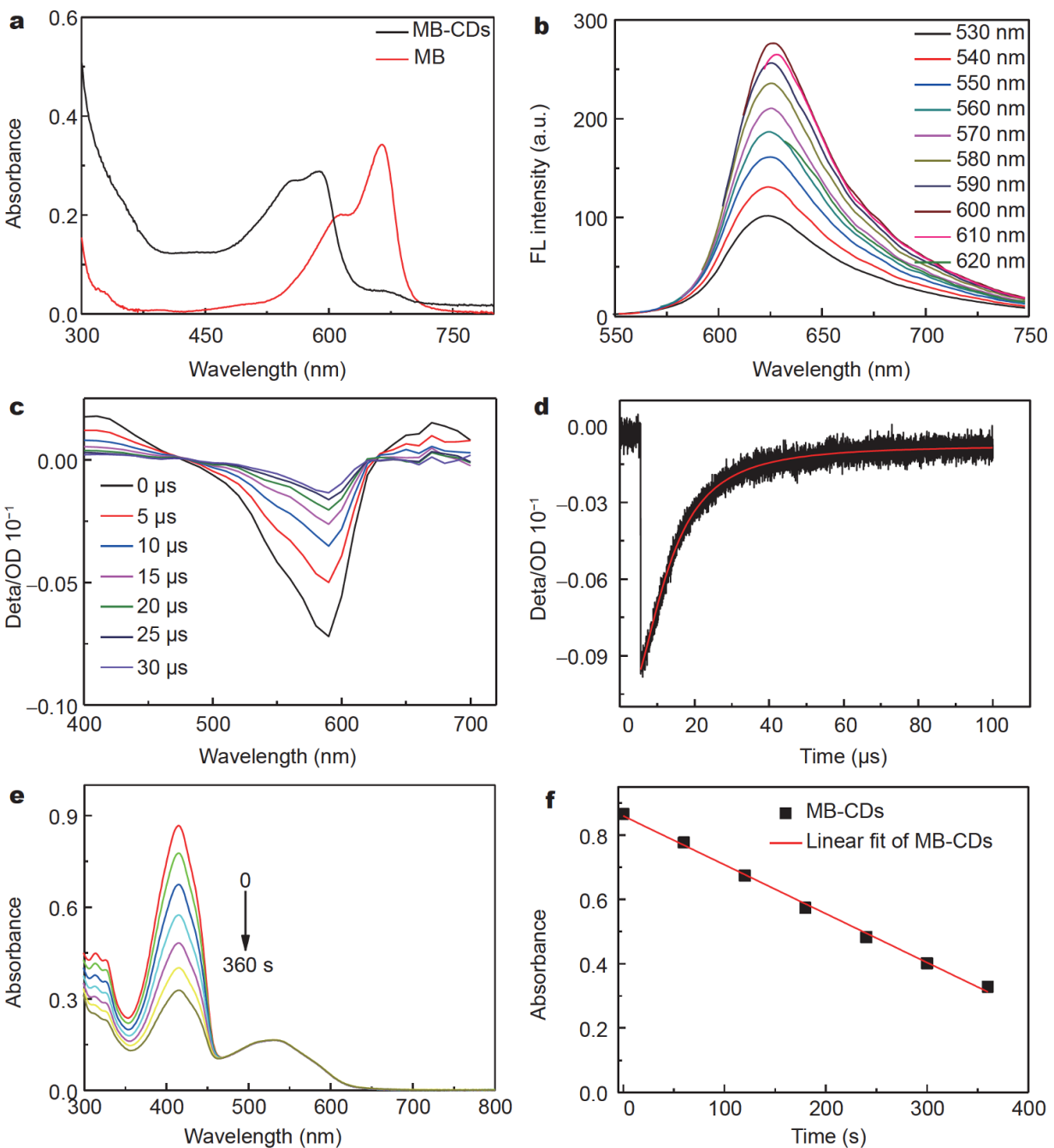

Figure 2 (a) UV-vis absorption spectra of MB and MB-CDs in water. (b) The fluorescence spectra of MB-CDs with different excitation wavelengths over 530-620 nm in water. (c) Nanosecond time-resolved transient absorption spectra of MB-CDs in deoxygenated water. (d) Dynamics spectra of MB-CDs in deoxygenated water. (e) Absorbance decrease of DPBF under 590-nm LED source irradiation in the presence of MB-CDs in DCM. (f) Linear fit of absorbance for DPBF at $415 \mathrm{~nm}$ for MB-CDs under light irradiation.

(control group) under the same conditions. The green fluorescence signal was significantly weaker after adding exogenous $\mathrm{NaN}_{3}$ (a reactive oxygen scavenger), indicating that the intracellular ROS had been quenched. Annexin V-FITC and PI were then used to evaluate treatmentinduced apoptosis. The green FITC fluorescence signal was detected in the cell membrane, indicating apoptosis. Meanwhile, the red fluorescence of PI was also observed in the nucleus, indicating cell death after PDT (Fig. S11). This result is consistent with the flow cytometry results (Fig. S12). Furthermore, Calcein-AM and PI, as a commercial live/dead cell-staining detection kit, were used to confirm the MB-CD PDT results. As shown in Fig. 3d, the green fluorescence signal of AM in the MCF-7 cells was hardly detected after cell exposure to light, a result consistent with that of few living cells. The PI channel showed strong red fluorescence, consistent with that of dead cells after PDT. At the same time, MCF-7 cells emitted a green fluorescence signal in the absence of light, revealing that the MB-CDs are essentially nontoxic in the dark. In line with the above-stated results, we showed that the MB-CDs have a high photodynamic efficiency.

\section{Photodynamic therapy experiments in vivo}

To further investigate the photodynamic properties of the $\mathrm{MB}-\mathrm{CDs}$ in vivo, we used $4 \mathrm{~T} 1$ tumor-bearing $\mathrm{Balb} / \mathrm{c}$ mice as a model. First, we injected a PBS buffer containing MB-CDs $\left(50 \mu \mathrm{g} \mathrm{mL}^{-1}\right)$ into the tumors of the above- 

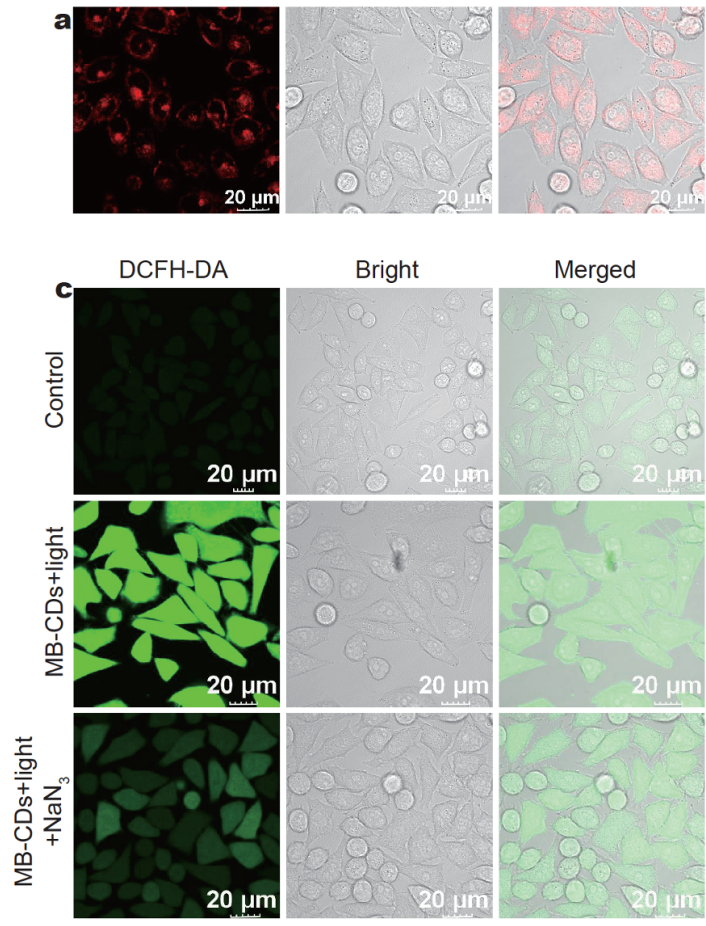

Merged
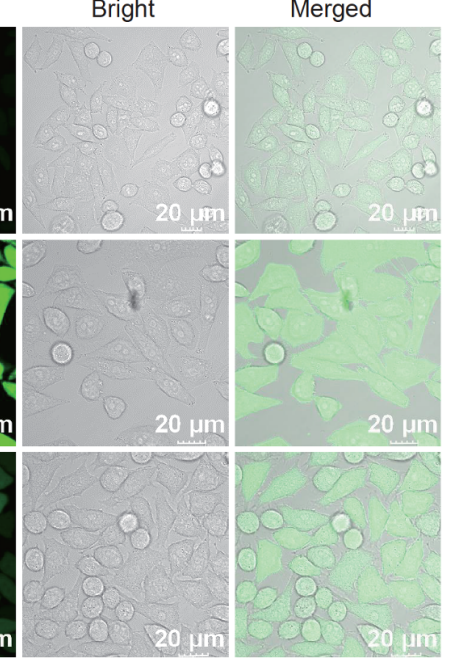

Figure 3 (a) The confocal fluorescent images of MCF-7 cells after incubation with MB-CDs. (b) The viability of MCF-7 cells incubated with a series of concentrations of MB-CDs before and after 590-nm LED source irradiation for 15 min. (c) Confocal microscopy imaging of MCF-7 cells with photo-induced intracellular ROS generation. (d) Confocal microscopy imaging of MCF-7 cells stained with Calcein-AM and PI before and after PDT treatment.

mentioned Balb/c mice to test whether or not the MB$\mathrm{CDs}$ accumulate and metabolize in the tumor. As shown in Fig. S13, tumors injected with the MB-CD solution show stronger fluorescence signals than other parts of the body. The $4 \mathrm{~T} 1$ tumor-bearing Balb/c mice were divided into four groups when the tumor volume reached $100 \mathrm{~mm}^{3}$ : (I) mice injected with PBS, (II) mice injected with PBS and LED irradiated $\left(590 \mathrm{~nm}, 50 \mathrm{~mW} \mathrm{~cm}^{-2}\right.$, $20 \mathrm{~min}$ ), (III) mice injected with MB-CDs, and (IV) mice injected with MB-CDs and LED irradiated (590 nm, $50 \mathrm{~mW} \mathrm{~cm}^{-2}, 20 \mathrm{~min}$ ). The tumor volumes and body weights of the mice in the four groups were measured and recorded every two days. As shown in Fig. 4a, the tumors increased in size significantly (about 9-12 times larger than the original) over time in the (I) PBS, (II) PBS + 590nm LED, and (III) MB-CD groups. The tumor growth in group (IV) was efficiently inhibited. The average tumor volume was reduced to $19.5 \%$ of the initial volume when irradiated by the $590-\mathrm{nm}$ LED. No significant weight losses were observed in all four groups during the entire treatment process. This indicated the limited biotoxicity of MB-CDs (Fig. 4b). In addition, we separated and measured the tumors of the four groups. The tumor volume in group (IV) was significantly smaller than the other three groups (Fig. 4c, d). Furthermore, the tumor slices after H\&E staining clearly show that the MB-CDs had destroyed the tumor tissue. As Fig. 4e shows, there is no obvious necrosis in the other three groups. To further examine the biocompatibility of MB-CDs, the main organs (heart, liver, spleen, lungs, and kidneys) of the mice were stained, revealing no detectable pathological damage. This indicated high biocompatibility (Fig. S14).

\section{Anti-reduction and DNA interference experiments in vitro} $\mathrm{MB}$ is readily reduced to uncharged and colorless leucoMB in vitro and in vivo by reductants $(\mathrm{NADH}$, glutathione, and so on, Fig. 5a) in a typical "blue bottle" experiment. Blue-to-colorless changes were observed when an $\mathrm{MB}$ solution $(1 \% \mathrm{NaOH})$ was shaken in the presence of glucose $(1 \%)$ as a reductant. In contrast to the $\sim 98 \%$ decrease in the absorption intensity observed for free $\mathrm{MB}$, the MB-CDs demonstrated almost no decline in intensity, suggesting they are anti-reductive (Fig. 5b). Furthermore, we prepared a solution consisting of NADH and diaphorase to replicate the reductive microenvironment in a solid tumor. As expected, the fluorescence signal of $\mathrm{MB}$ in the $\mathrm{NADH}+$ diaphorase containing the experimental group was rapidly quenched to $8 \%$ of its 

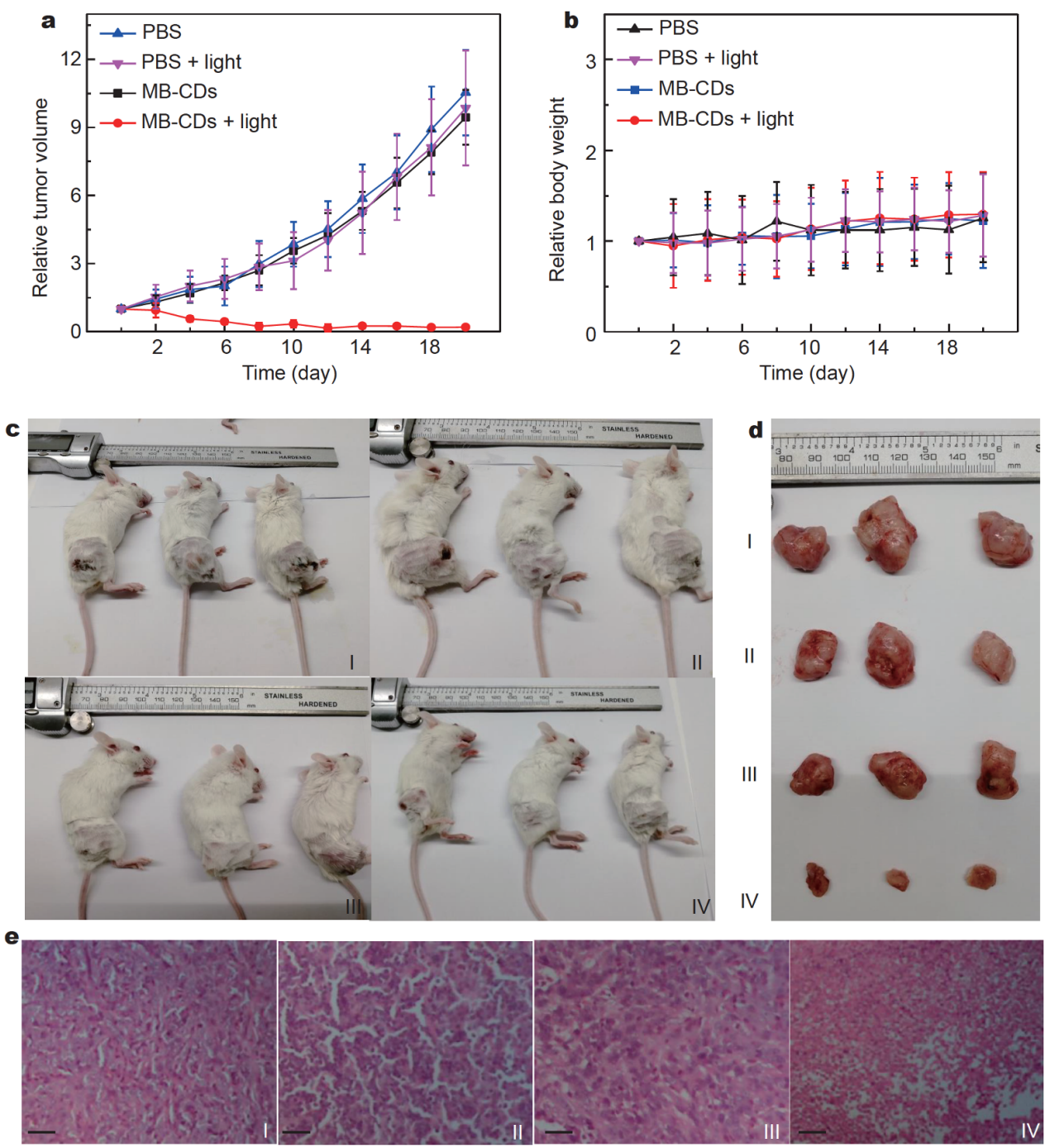

Figure 4 (a) Tumor growth curves for groups (I)-(IV). (b) Relative changes in body weight for groups (I)-(IV). (c) Photos of 4T1 tumor-bearing mice after PDT treatment for $20 \mathrm{~d}$ in groups (I)-(IV). (d) Photos of the tumors after PDT treatment for $20 \mathrm{~d}$ in groups (I)-(IV). (e) H\&E stained tumor sections for groups (I)-(IV) (scale bars: $50 \mu \mathrm{m}$ ).

original value within $1 \mathrm{~min}$, whereas after $50 \mathrm{~min}$, the signal recovered slightly due to the reductant depletion (pink triangles, Fig. 5c and Fig. S15). The MB-CDs exhibited a transitory decrease in fluorescence intensity (to $82 \%)$ and then recovered to nearly $100 \%$ of the original value within $15 \mathrm{~min}$ and remained stable thereafter (red points, Fig. $5 c$ and Fig. S16). These experimental results show that the MB-CDs are very anti-reducing and maintain good photodynamic activity in a reducing atmosphere compared with MB.

Next, we investigated how NADH and diaphorase affect the efficiency of MB and MB-CDs for PDT in MTT experiments. As shown in Fig. 5d, the cell viability was above $80 \%$ after introducing increasing amounts of $\mathrm{MB}$, indicating that $\mathrm{MB}$ is non-biotoxic (black bars) in the absence of light. Cell viability slightly changed after introducing NADH and diaphorase to the MCF-7 cells, which shows they are safe (backslashed bars). As expected, the cell viability of the $\mathrm{MB}$ group decreased rapidly $(100 \% \rightarrow 73 \% \rightarrow 33 \% \rightarrow 2 \% \rightarrow 1 \%)$ when exposed to light (slashed bars). However, the presence of NADH and diaphorase significantly retarded the rate of cell death $(100 \% \rightarrow 94 \% \rightarrow 56 \% \rightarrow 45 \% \rightarrow 26 \%$, blank bars $)$. For example, $0.1 \mu \mathrm{mol} \mathrm{L}^{-1} \mathrm{MB}$ killed $27 \%$ of the cells when exposed to light; however, the presence of the NADH and diaphorase supplied a reductive environment and restricted $\mathrm{MB}$ effects completely. A cell viability of around $2 \%$ was achieved when $1.5 \mu \mathrm{mol} \mathrm{L}^{-1}$ of $\mathrm{MB}$ was used 
$\mathbf{a}$

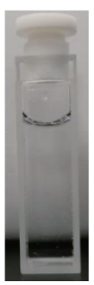

b
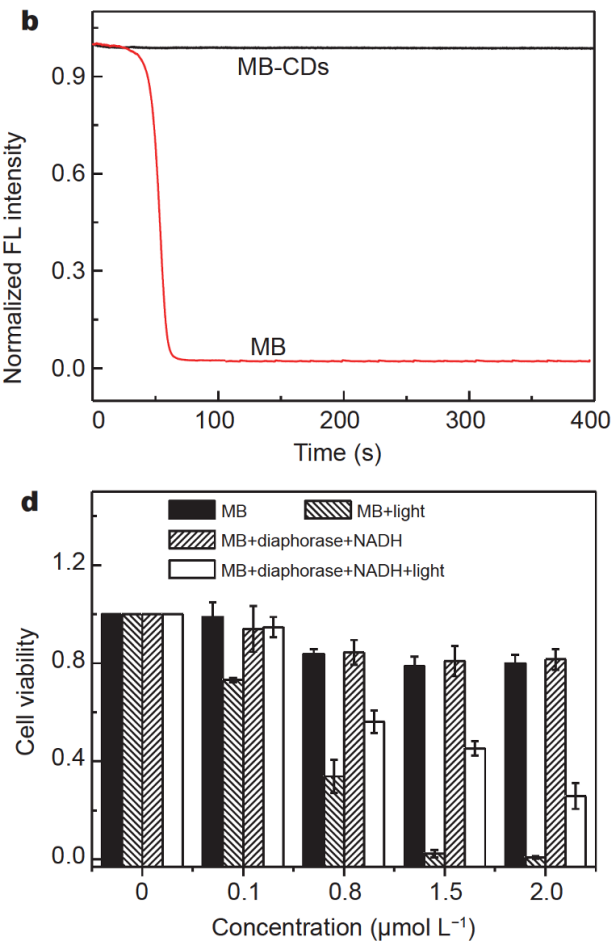
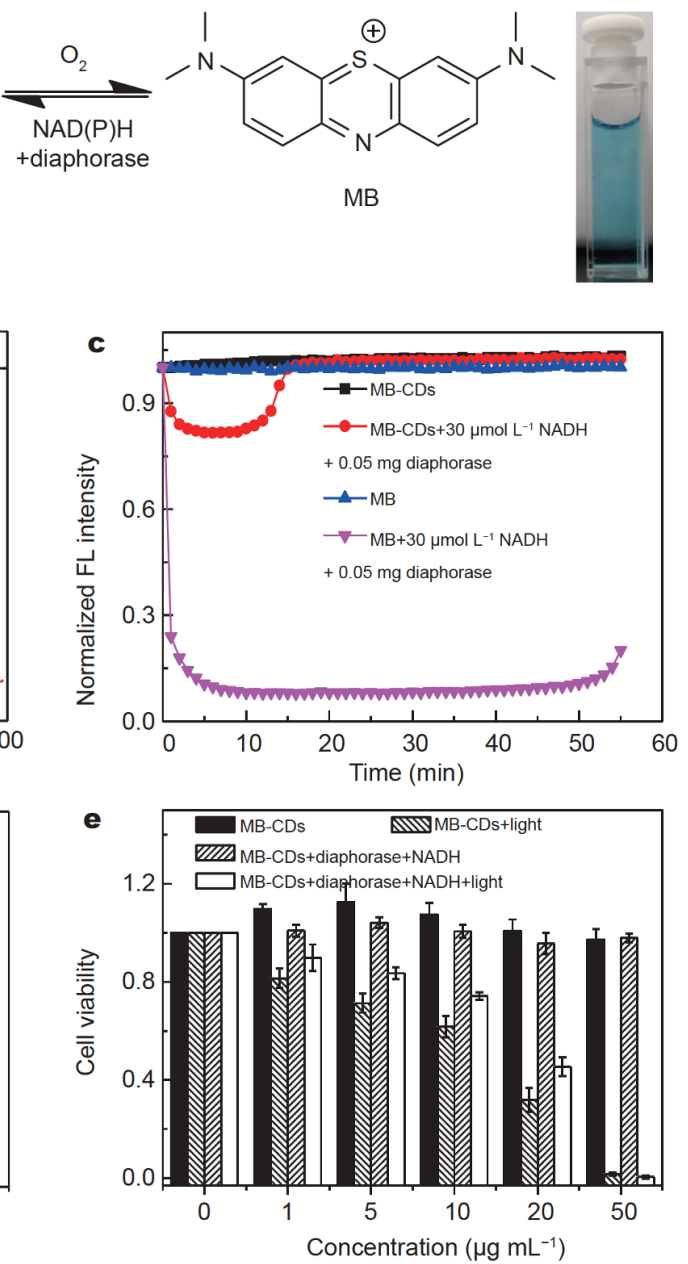

Figure 5 (a) Schematic diagram showing the chemistry of $\mathrm{MB}$ in the reductive microenvironment of diaphorase and NADH. (b) Blue bottle experiment (glucose $+\mathrm{NaOH}$ ): fluorescence intensities of MB-CDs and $\mathrm{MB}$ at $680 \mathrm{~nm}$ as functions of time. (c) Fluorescence intensities of MB and MB-CDs as functions of time in the presence or absence of diaphorase and NADH $\left([\mathrm{MB}]=0.3 \mu \mathrm{mol} \mathrm{L}{ }^{-1},[\mathrm{MB}-\mathrm{CDs}]=100 \mu \mathrm{g} \mathrm{mL}{ }^{-1}\right.$, $[\mathrm{NADH}]=$ $30 \mu \mathrm{mol} \mathrm{L}{ }^{-1}$, [diaphorase] $=0.05 \mathrm{mg}$ ). Viabilities of MCF-7 cells incubated with various concentrations of (d) MB and (e) MB-CDs with and without irradiation with light and in the absence and presence of diaphorase and NADH.

under light irradiation, while around $45 \%$ of the cells remained viable for the $\mathrm{MB}+\mathrm{NADH}+$ diaphorase group under the same conditions. These results show that the reductive microenvironment significantly affects the PDT effectiveness of $\mathrm{MB}$.

As shown in Fig. 5e, $100 \%$ of the cells remained viable using the MB-CDs, even at $50 \mu \mathrm{g} \mathrm{mL}^{-1}$, clearly showing that the MB-CDs are less biotoxic (black bars) than $\mathrm{MB}$ $(80 \%)$ in the absence of light. No obvious change in cell viability was observed after introducing $\mathrm{NADH}$ and diaphorase to the MCF-7 cells (backslashed bars). The cell viability of the MB-CD group decreased significantly $(100.0 \% \rightarrow 81.4 \% \rightarrow 71.2 \% \rightarrow 61.7 \% \rightarrow 31.8 \% \rightarrow 1.5 \%$, slashed bars) with increasing concentration $(0,1,5,10,20$, and $50 \mu \mathrm{g} \mathrm{mL}^{-1}$ ) during the photodynamic process when ir- radiated with light. However, cell viability was affected little by introducing NADH and diaphorase. This result highlights the anti-reductive properties of the MB-CDs.

$\mathrm{MB}$ can interact with biomolecules, such as DNA (Fig. 6a). However, to the best of our knowledge, no study has examined how MB-DNA interactions affect PDT. As shown in Fig. 6b, the absorption of $\mathrm{MB}$ at $665 \mathrm{~nm}$ decreased significantly $(100.0 \% \rightarrow 75.9 \% \rightarrow 60.4 \% \rightarrow 51.1 \% \rightarrow$ $44.8 \% \rightarrow 40.8 \%)$ with the gradual addition of dsDNA $(0,1$, 2, 3, 4, $5 \mu \mathrm{g} \mathrm{mL}^{-1}$ ), while that of the MB-CDs remained stable even in the presence of $50 \mu \mathrm{g} \mathrm{mL}^{-1}$ of dsDNA $(100.0 \% \rightarrow 91.9 \%)$. Hypochromicity is observed because $\mathrm{MB}$ interacts with DNA through intercalative binding. According to the Grotthuss-Draper law (i.e., the photochemical activation law), only light absorbed by the 
a dsDNA

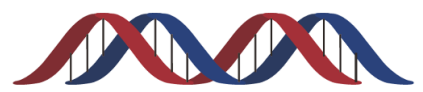

$\mathrm{MB}$

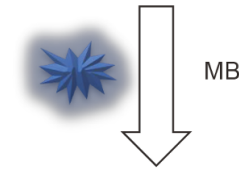

dsDNA/MB
complexes
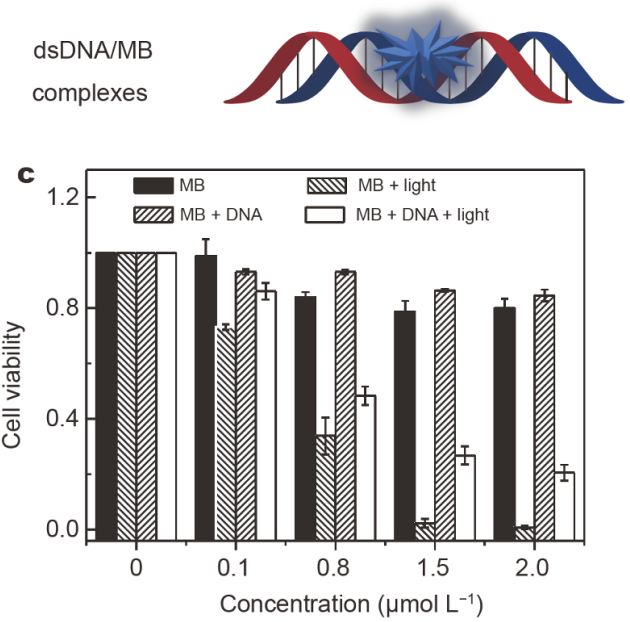
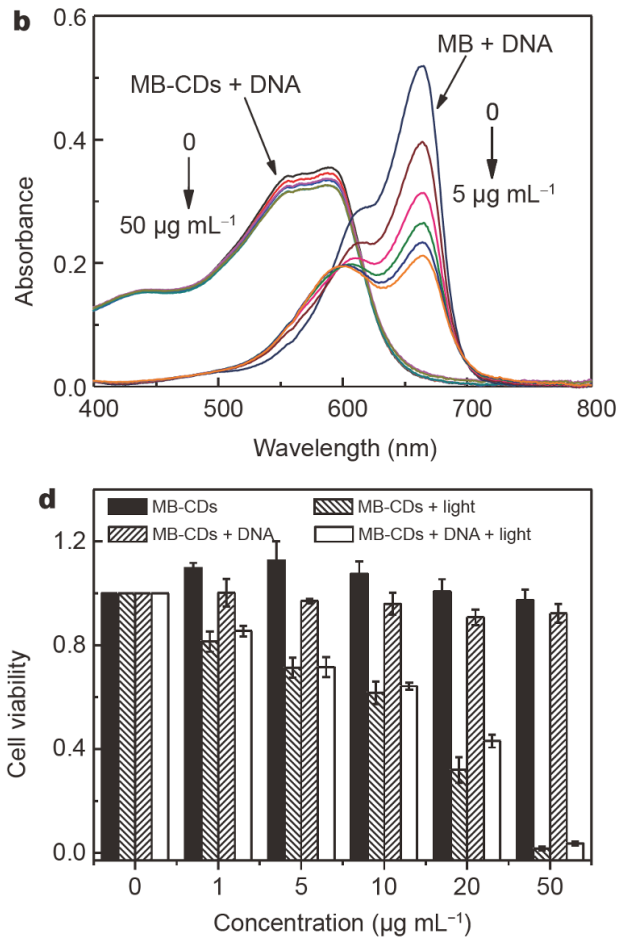

Figure 6 (a) Schematic diagram of an MB-DNA complex. (b) Absorption spectra of MB and MB-CDs in the absence and presence of various concentrations of DNA $\left(0-5 \mu \mathrm{gL}^{-1}\right)$ in water at room temperature. MTT data for (c) MB and (d) MB-CDs with and without irradiation with light and in the absence and presence of DNA $\left(5 \mu \mathrm{g} \mathrm{m}^{-1}\right)$.

photosensitizer can be used in PDT. Therefore, binding between MB and DNA decreases the PDT effectiveness, as shown by the MTT results. Fig. $6 \mathrm{c}$ reveals that MB is not much biotoxic (black bars) in the absence of light. In the absence of light, DNA introduction increased cell viability by $10 \%-20 \%$ (backslashed bars), indicating interactions between $\mathrm{MB}$ and dsDNA. The cell death rate for the MB + DNA group was obviously lower than that of the MB group when irradiated by light. This trend became more obvious with increasing $\mathrm{MB}$ concentration. As shown in Fig. 6d, almost $100 \%$ cell viability was observed for the MB-CDs, both in the presence and absence of DNA (black bars and slashed bars). Most importantly, we observed almost the same changes in cell viability for the MB-CDs in the absence and presence of DNA when irradiated by light (backslashed bars and blank bars). The different behavior of MB and the MB-CDs with DNA stems from their different zeta potentials. The positively charged $\mathrm{MB}$ was transformed into the negatively charged MB-CDs (zeta potential: $-3.63 \mathrm{mV}$; Fig. S2) during the hydrothermal reactions. Therefore, both steric effects and electrostatic repulsion between the MB-CDs and dsDNA inhibit their interactions.

\section{CONCLUSIONS}

In summary, we used a hydrothermal reaction with $\mathrm{MB}$ as the carbon source to synthesize unique MB-CDs that are very biocompatible and nontoxic. Importantly, the MB-CDs inherited the excellent photosensitive properties of $\mathrm{MB}$ and evolved to include additional capacities against reduction, aggregation, and DNA interference that heavily affect MB performance during solid-tumor PDT. The MB-CDs proved effective for PDT in both in vitro and in vivo experiments. Tumor sizes were reduced to below $20 \%$ of their original volumes upon only once irradiation, compared with more than 9-fold increase of tumor size without light treatment. Currently, a combination of PDT and surgery is the most feasible strategy, in which PDT kills residual cells after visible tumors have been surgically removed. The MB-CDs developed in this work, which require only once irradiation, show significant potential for tumor treatment applications.

\section{Received 4 December 2020; accepted 21 January 2021;} published online 9 April 2021

1 Li X, Kim J, Yoon J, et al. Cancer-associated, stimuli-driven, turn on theranostics for multimodality imaging and therapy. Adv Ma- 
ter, 2017, 29: 1606857

2 Cui D, Huang J, Zhen X, et al. A semiconducting polymer nanoprodrug for hypoxia-activated photodynamic cancer therapy. Angew Chem Int Ed, 2019, 58: 5920-5924

3 Han HH, Wang CZ, Zang Y, et al. Supramolecular core-glycoshell polythiophene nanodots for targeted imaging and photodynamic therapy. Chem Commun, 2017, 53: 9793-9796

4 Tian B, Wang C, Zhang S, et al. Photothermally enhanced photodynamic therapy delivered by nano-graphene oxide. ACS Nano, 2011, 5: 7000-7009

5 Sun Q, He F, Sun C, et al. Honeycomb-satellite structured pH/ $\mathrm{H}_{2} \mathrm{O}_{2}$-responsive degradable nanoplatform for efficient photodynamic therapy and multimodal imaging. ACS Appl Mater Interfaces, 2018, 10: 33901-33912

6 Wang Z, Jia T, Sun Q, et al. Construction of Bi/phthalocyanine manganese nanocomposite for trimodal imaging directed photodynamic and photothermal therapy mediated by $808 \mathrm{~nm}$ light. Biomaterials, 2020, 228: 119569

7 Li X, Lee S, Yoon J. Supramolecular photosensitizers rejuvenate photodynamic therapy. Chem Soc Rev, 2018, 47: 1174-1188

8 Zhou Z, Song J, Nie L, et al. Reactive oxygen species generating systems meeting challenges of photodynamic cancer therapy. Chem Soc Rev, 2016, 45: 6597-6626

9 Li J, Rao J, Pu K. Recent progress on semiconducting polymer nanoparticles for molecular imaging and cancer phototherapy. Biomaterials, 2018, 155: 217-235

$10 \mathrm{Wu} \mathrm{X}$, Sun $\mathrm{X}$, Guo Z, et al. In vivo and in situ tracking cancer chemotherapy by highly photostable NIR fluorescent theranostic prodrug. J Am Chem Soc, 2014, 136: 3579-3588

11 Kumar R, Shin WS, Sunwoo K, et al. Small conjugate-based theranostic agents: An encouraging approach for cancer therapy. Chem Soc Rev, 2015, 44: 6670-6683

12 Allison RR, Downie GH, Cuenca R, et al. Photosensitizers in clinical PDT. Photodiagn Photodyn Ther, 2004, 1: 27-42

13 Zhu H, Li J, Qi X, et al. Oxygenic hybrid semiconducting nanoparticles for enhanced photodynamic therapy. Nano Lett, 2018, 18: 586-594

14 Cheng L, Wang C, Feng L, et al. Functional nanomaterials for phototherapies of cancer. Chem Rev, 2014, 114: 10869-10939

15 Sun Q, He F, Bi H, et al. An intelligent nanoplatform for simultaneously controlled chemo-, photothermal, and photodynamic therapies mediated by a single NIR light. Chem Eng J, 2019, 362: 679-691

16 Gonzalez-Bejar M, Montes-Navajas P, Garcia H, et al. Methylene blue encapsulation in cucurbit[7]uril: Laser flash photolysis and near-IR luminescence studies of the interaction with oxygen. Langmuir, 2009, 25: 10490-10494

17 Chen Y, Zheng W, Li Y, et al. Apoptosis induced by methyleneblue-mediated photodynamic therapy in melanomas and the involvement of mitochondrial dysfunction revealed by proteomics. Cancer Sci, 2008, 99: 2019-2027

18 Tardivo JP, Del Giglio A, de Oliveira CS, et al. Methylene blue in photodynamic therapy: From basic mechanisms to clinical applications. Photodiagn Photodyn Ther, 2005, 2: 175-191

19 Orth K, Beck G, Genze F, et al. Methylene blue mediated photodynamic therapy in experimental colorectal tumors in mice. J Photochem Photobiol B-Biol, 2000, 57: 186-192

20 Blázquez-Castro A, Stockert JC, Sanz-Rodríguez F, et al. Differential photodynamic response of cultured cells to methylene blue and toluidine blue: Role of dark redox processes. Photochem
Photobiol Sci, 2009, 8: 371-376

21 Tang W, Xu H, Park EJ, et al. Encapsulation of methylene blue in polyacrylamide nanoparticle platforms protects its photodynamic effectiveness. Biochem Biophys Res Commun, 2008, 369: 579-583

22 Nuñez SC, Yoshimura TM, Ribeiro MS, et al. Urea enhances the photodynamic efficiency of methylene blue. J Photochem Photobiol B-Biol, 2015, 150: 31-37

23 Nogueira JJ, González L. Molecular dynamics simulations of binding modes between methylene blue and DNA with alternating GC and AT sequences. Biochemistry, 2014, 53: 2391-2412

24 Nogueira JJ, Roßbach S, Ochsenfeld C, et al. Effect of DNA environment on electronically excited states of methylene blue evaluated by a three-layered QM/QM/MM ONIOM scheme. J Chem Theor Comput, 2018, 14: 4298-4308

25 Gao P, Wang J, Zheng M, et al. Lysosome targeting carbon dotsbased fluorescent probe for monitoring $\mathrm{pH}$ changes in vitro and in vivo. Chem Eng J, 2020, 381: 122665

26 Li J, Yang S, Deng Y, et al. Emancipating target-functionalized carbon dots from autophagy vesicles for a novel visualized tumor therapy. Adv Funct Mater, 2018, 28: 1800881

$27 \mathrm{Du}$ J, Xu N, Fan J, et al. Carbon dots for in vivo bioimaging and theranostics. Small, 2019, 15: 1805087

28 Ren X, Liang W, Wang P, et al. A new approach in functionalization of carbon nanoparticles for optoelectronically relevant carbon dots and beyond. Carbon, 2019, 141: 553-560

29 Jiang K, Gao X, Feng X, et al. Carbon dots with dual-emissive, robust, and aggregation-induced room-temperature phosphorescence characteristics. Angew Chem Int Ed, 2020, 59: 1263-1269

30 Li C, Zhang K, Yao C, et al. Altering sub-cellular location for bioimaging by engineering the carbon based fluorescent nanoprobe. Sci China Mater, 2019, 62: 1496-1504

31 Sun S, Chen J, Jiang K, et al. Ce6-modified carbon dots for multimodal-imaging-guided and single-NIR-laser-triggered photothermal/photodynamic synergistic cancer therapy by reduced irradiation power. ACS Appl Mater Interfaces, 2019, 11: 5791-5803

32 Zhang Q, Sun X, Ruan H, et al. Production of yellow-emitting carbon quantum dots from fullerene carbon soot. Sci China Mater, 2017, 60: 141-150

33 Zhu S, Meng Q, Wang L, et al. Highly photoluminescent carbon dots for multicolor patterning, sensors, and bioimaging. Angew Chem Int Ed, 2013, 52: 3953-3957

34 Ge J, Lan M, Zhou B, et al. A graphene quantum dot photodynamic therapy agent with high singlet oxygen generation. Nat Commun, 2014, 5: 4596

35 He H, Zheng X, Liu S, et al. Diketopyrrolopyrrole-based carbon dots for photodynamic therapy. Nanoscale, 2018, 10: 10991-10998

36 Jia Q, Ge J, Liu W, et al. A magnetofluorescent carbon dot assembly as an acidic $\mathrm{H}_{2} \mathrm{O}_{2}$-driven oxygenerator to regulate tumor hypoxia for simultaneous bimodal imaging and enhanced photodynamic therapy. Adv Mater, 2018, 30: 1706090

37 Miao X, Yan X, Qu D, et al. Red emissive sulfur, nitrogen codoped carbon dots and their application in ion detection and theraonostics. ACS Appl Mater Interfaces, 2017, 9: 18549-18556

38 Lan M, Zhao S, Wu S, et al. Optically tunable fluorescent carbon nanoparticles and their application in fluorometric sensing of copper ions. Nano Res, 2019, 12: 2576-2583

39 Li Y, Dong H, Tao Q, et al. Enhancing the magnetic relaxivity of MRI contrast agents via the localized superacid microenvironment of graphene quantum dots. Biomaterials, 2020, 250: 120056

$40 \mathrm{Xu} \mathrm{N}, \mathrm{Du}$ J, Yao Q, et al. Precise photodynamic therapy: Pene- 
trating the nuclear envelope with photosensitive carbon dots. Carbon, 2020, 159: 74-82

41 Liu J, Geng Y, Li D, et al. Deep red emissive carbonized polymer dots with unprecedented narrow full width at half maximum. Adv Mater, 2020, 32: 1906641

42 Xia C, Zhu S, Feng T, et al. Evolution and synthesis of carbon dots: From carbon dots to carbonized polymer dots. Adv Sci, 2019, 6: 1901316

43 Shamsipur M, Barati A, Karami S. Long-wavelength, multicolor, and white-light emitting carbon-based dots: Achievements made, challenges remaining, and applications. Carbon, 2017, 124: 429472

44 Zuo P, Lu X, Sun Z, et al. A review on syntheses, properties, characterization and bioanalytical applications of fluorescent carbon dots. Microchim Acta, 2016, 183: 519-542

45 Chung S, Revia RA, Zhang M. Graphene quantum dots and their applications in bioimaging, biosensing, and therapy. Adv Mater, 2019, 1904362

46 Ristic BZ, Milenkovic MM, Dakic IR, et al. Photodynamic antibacterial effect of graphene quantum dots. Biomaterials, 2014, 35: 4428-4435

47 Zhao J, Wu W, Sun J, et al. Triplet photosensitizers: From molecular design to applications. Chem Soc Rev, 2013, 42: 5323-5351

48 Xu F, Li H, Yao Q, et al. Hypoxia-activated NIR photosensitizer anchoring in the mitochondria for photodynamic therapy. Chem Sci, 2019, 10: 10586-10594

Acknowledgements This work was financially supported by the National Natural Science Foundation of China (NSFC, 21822804, 21676047, 21421005, 21878039, and 21925802) and NSFC-Liaoning United Fund (U1908202 and U1608222).

Author contributions This work was finished through the contributions of all authors. The design and synthesis of the carbon dots used in this work, most of the tests in vivo and in vitro were contributed by $\mathrm{Xu} \mathrm{N}$, and $\mathrm{Gu} \mathrm{Q}$ was in charge of cell culture in the experiment. The ToC and Scheme 1, and TEM tests were completed by Ge H, and Dr. Long $S$ gave supports to the intriplet lifetime tests. Prof. Fan J and Dr. Sun W offered very important suggestions and helped on how to improve experiments. Prof. Du J and Prof. Peng X directed the whole process in this work and revised the manuscript. All authors have given approval to the final version of this manuscript.

Conflict of interest The authors declare that they have no conflict of interest.

Supplementary information online version of the paper.

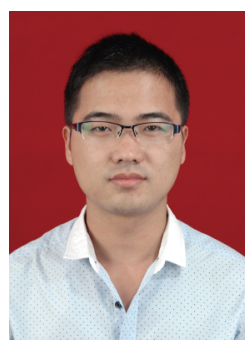

Ning $\mathrm{Xu}$ received his $\mathrm{BE}$ degree from Anhui University of Science \& Technology in 2015. Currently, he is pursuing his $\mathrm{PhD}$ degree under the supervision of Prof. Xiaojun Peng at Dalian University of Technology (DUT). His current research interests include synthesis and applications of carbon dots.

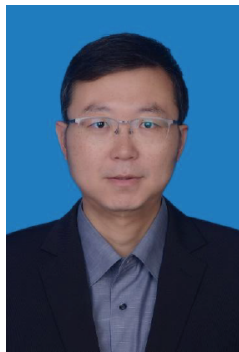

Jianjun Du is a professor at the State Key Laboratory of Fine Chemicals at DUT. He received his $\mathrm{BSc}$ and $\mathrm{PhD}$ degrees in applied chemistry from DUT in 2004 and 2010, respectively. After his postdoctoral fellowship work at Nanyang Technological University (Singapore) in 20102013, he started his independent research career at DUT. His research is focused on functional dyes and nanoparticles for sensing, imaging and theranostics.

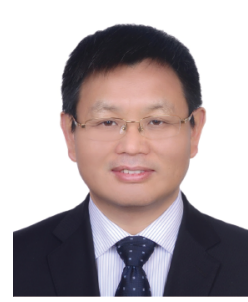

Xiaojun Peng received his PhD degree in 1990 at DUT. After completing postdoctoral research at Nankai University (China), he has worked at DUT since 1992. In 2001 and 2002, he was a visiting scholar at Stockholm University and Northwestern University (USA). Currently he is director of the State Key Laboratory of Fine Chemicals at DUT. His research interests include functional dyes for fluorescent bioimaging/ labeling and digital printing/recording.

\section{基于亚甲基蓝制备的抗还原、抗聚集和抗DNA干 扰的光动力治疗碳点}

\author{
徐宁 ${ }^{1}$, 谷泉泳 ${ }^{1}$, 杜健军 ${ }^{1,2^{*}}$, 葛浩英 ${ }^{1}$, 龙飒然 ${ }^{1,2}$, 孙文 ${ }^{1,2}$, \\ 㚞江莉 ${ }^{1,2}$, 彭孝军 ${ }^{1}$
}

摘要 光动力疗法 $(\mathrm{PDT})$ 是一种重要的癌症治疗手段, 亚甲基蓝 $(\mathrm{MB})$ 作为一种有效的光敏剂被广泛应用于光动力治疗. 然而, $\mathrm{MB}$ 在体内由于聚集以及与DNA结合而导致减色效应. 同时, MB在实 体瘤的低氧环境中容易被还原为无色的还原态leucoMB, 从而丧失 光动力能力. 因此, 我们以 $\mathrm{MB}$ 作为碳源, 制备碳点MB-CDs. MB$\mathrm{CDs}$ 不仅继承了 $\mathrm{MB}$ 的PDT能力, 而且表现出良好的生物相容性和 低毒性. 重要的是, MB-CDs具有出色的抗还原、抗聚集和抗DNA 干扰的能力, 并且MB-CDs在体外和体内均表现出良好的PDT活性, 仅用 $590 \mathrm{~nm} \mathrm{LED}$ 光源照射一次, 即可使肿瘤体积减小到原始体积 的 $20 \%$ 以下. 